\title{
Guanidinoacetate methyltransferase deficiency
}

INSERM

\section{Source}

INSERM. (1999). Orphanet: an online rare disease and orphan drug data base.

Guanidinoacetate methyltransferase deficiency. ORPHA:382

Guanidinoacetate methyltransferase (GAMT) deficiency is a creatine deficiency syndrome characterized by global developmental delay/intellectual disability (DD/ID), prominent speech delay, autistic/hyperactive behavioral disorders, seizures, and various types of pyramidal and/or extra-pyramidal manifestations. 